\title{
Imaging dermal blood flow through the intact rat skin with an optical clearing method
}

\author{
Dan Zhu \\ Jing Wang \\ Zhongwei Zhi \\ Xiang Wen \\ Qingming Luo \\ Huazhong University of Science and Technology \\ Britton Chance Center for Biomedical Photonics \\ Wuhan National Laboratory for Optoelectronics \\ Wuhan 430074, China
}

\begin{abstract}
Noninvasive detection of skin microcirculation is very significant for clinical diagnosis and therapy of peripheral vascular disease. In this study, an optical clearing method enables a laser speckle contrast imaging ( $\mathrm{LSCl}$ ) technique to image dermal blood flow through intact rat skin. The optical clearing effect of rat skin in vitro caused by a mixture of PEG-400 and Thiazone is evaluated by a commercially available spectrophotometer with an integrating sphere. A LSCI system is applied to image the dermal blood flow of in vivo rat skin after topical treatment of the agents. The results show that the reduced scattering coefficient $(630 \mathrm{~nm})$ is down to $60 \%$ of the initial value after $40 \mathrm{~min}$ of topical treatment of the mixture on skin in vitro. In vivo experimental results show that a 12-min treatment of the mixture on the epidermis can make the skin transparent and dermal vessels can be observed, while treatment with pure PEG-400 cannot. The process of skin optical clearing lasts, and the skin can be recovered by treatment of saline solution. Because optical clearing decreases the temporal contrast, dermal blood flow can be obtained by the laser speckle contrast imaging technique. ๑ 2010 Society of Photo-Optical Instrumentation Engineers. [DOI: 10.1117/1.3369739]
\end{abstract}

Keywords: optical clearing; laser speckle contrast imaging; dermal blood flow monitoring; optical properties; Thiazone.

Paper 09246PRR received Jun. 12, 2009; revised manuscript received Jan. 23, 2010; accepted for publication Jan. 26, 2010; published online Apr. 23, 2010.

\section{Introduction}

Impairment of skin microcirculation is one of the main features in advanced clinical stages of peripheral vascular disease. Recently, some noninvasive methods have been available to investigate skin microcirculation in clinical settings as well as improve disease management, i.e., capillaroscopy allows the investigation of skin capillary morphology and density, laser Doppler fluxmetry allows real-time assessment of perfusion of skin volume, and transcutaneous measurement of oxygen tension provides continuous information about skin oxygenation. ${ }^{1}$ However, none of them can provide both structural and functional information of dermal vessels simultaneously. In contrast, laser speckle contrast imaging (LSCI), a full-field optical technique, can produce a two-dimensional map of blood flow with high spatio-temporal resolution, ${ }^{2-7}$ which plays an important role in studying cerebral blood flow, ${ }^{7,8}$ mesentery microcirculation, ${ }^{9}$ etc. Unfortunately, it suffers from a limited penetration depth of light in turbid tissues, as the previous investigations were restricted within the image of blood flow in transparent tissues.

Actually, immersion in cedar-wood oil or vaseline oil has been traditionally used in classical microscopy and capillaroscopy to improve light penetration. ${ }^{10}$ In 1997, Tuchin et al. proposed the tissue optical control technique, ${ }^{11}$ and then rela-

Address all correspondence to: Qingming Luo, Huazhong University of Science and Technology, Britton Chance Center for Biomedical Photonics, Wuhan National Laboratory for Optoelectronics, Wuhan, 430074, China. Tel: 86-2787792033; Fax: 86-27-87792034; E-mail: qluo@mail.hust.edu.cn tive investigations attracted more and more attention. ${ }^{12}$ The immersion solutions are called optical clearing agents (OCAs), and the optical control technique is also called the tissue optical clearing technique. ${ }^{12}$ However, the outermost layer of the skin, the stratum corneum (SC), presents a significant barrier for the most topical application of OCAs and is hence responsible for the poor optical clearing effect. To reduce the barrier function of SC, numerous methods such as physical methods to remove SC (photothermal, ${ }^{13}$ ultrasound, ${ }^{14}$ sand paper, ${ }^{15}$ and microneedle arrays ${ }^{16}$ ) and chemical penetration enhancers (DMSO, ${ }^{17}$ Azone,${ }^{18}$ Thiazone ${ }^{19,20}$ ) have been proposed, which have been shown to enhance the skin in vitro permeability of OCAs to a certain extent. Nevertheless, physical methods for SC removal always result in some damage, the commonly used penetration enhancer DMSO has potential toxicity, and the penetration enhancing effect of Azone is not ideal. $^{20}$ Thiazone, as a new penetration enhancer, has higher enhancing effect, but is used to study the optical clearing of in vitro skin. ${ }^{19,20}$ In fact, metabolism prevents OCAs assembling in local tissue, which makes the optical clearing of skin in vivo more difficult to achieve than that of skin in vitro. Hence, hypodermic injection became a common method to improve optical clearing of skin in vivo, but it has always been associated with some degree of scathe to skin or even the blockage of blood vessels. ${ }^{21,22}$ Therefore, previous studies on monitoring skin blood flow with LSCI were still based on an invasive dorsal skin flap window model. ${ }^{23}$

$1083-3668 / 2010 / 15(2) / 026008 / 7 / \$ 25.00$ ○ 2010 SPIE 
In view of these facts, the purpose of this work is to develop a noninvasive method to access structure and function of the dermal blood vessels through the intact rat skin. Here we make an attempt on topical application of a new chemical penetration enhancer-thiazone mixed with PEG-400- to improve the effect of skin optical clearing. And some control experiments with pure PEG-400 or saline were conducted to evaluate the degree of improvement of the penetration enhancer. To quantitatively demonstrate the effectiveness of the optical clearing method, a commercially available spectrophotometer with an integrating sphere is used to measure the changes in optical properties of rat skin in vitro before and after treatment of saline, PEG-400, and the mixture of PEG400 and thiazone. Furthermore, the LSCI technique is applied to image the dermal blood flow of in vivo rat skin after topical treatment of those agents, and then the recovery of skin induced by saline is observed. The laser speckle temporal contrast analysis (LSTCA) method is applied to calculate velocity, because it can significantly suppress the influence of the static speckle pattern, which is produced by the stationary superficial scattering tissue on the visualization of blood flow. ${ }^{6,7}$

\section{Materials and Methods}

\subsection{Chemical Agents}

In this work, two chemical agents, thiazone (Heming Trading Company Limited, Guangzhou, China) and PEG-400 (Kemiou Reagent Development Corporation, Tianjing, China), are adopted.

Thiazone [benzisothiazol-3(2H)-one-2-butyl-1, 1-dioxide] is a kind of new skin penetration enhancer with a nonirritant and is free of immune responses to skin. ${ }^{24}$ It is a white crystalline at room temperature, and can be melted at $40{ }^{\circ} \mathrm{C}$. Its mechanism of penetration enhancing is similar to Azone, but the penetration enhancing effect is almost two times higher than Azone, and is considered to be an ideal substitute of Azone. PEG-400, as a kind of optical clearing agent, is widely used in the study of skin optical clearing. ${ }^{12}$ PEG-400 has a refractive index of 1.47 , and can be miscible with Thiazone. ${ }^{19,20}$

To optimize the enhancing effect, Thiazone was usually mixed with chemical agents as a volume percent of 0.5 to $10 \%{ }^{25}$ Before the experiment, Thiazone was heated to melt at 40 to $45^{\circ} \mathrm{C}$ and then mixed with PEG-400 as a volume ratio of 1:9; after that, the mixture solution was kept at room temperature $\left(20\right.$ to $\left.25^{\circ} \mathrm{C}\right)$. The mixture has been able to keep in a liquid state when the temperature is higher than $15^{\circ} \mathrm{C}$.

\subsection{Preparation of Model}

Male SD rats, age-matched at 3 to 4 weeks old $(n=37$, mean weight $=80$ to $100 \mathrm{~g}$ ), were obtained from Hubei Health and Epidemic Prevention Station (Wuhan, China). Before the experiment, rats were anaesthetized with a cocktail of $2 \%$ $\alpha$-chloralose and $10 \%$ urethane $(0.8-\mathrm{m} 1 / 100$-g weight $)$ via intraperitoneal injection. The dorsal hair was shaved, and the residue hair was removed using depilatory cream (Bamboo \& Salt moistening depilatory cream, LULANJINA, China). The skin was then stripped 3 to 4 times with an adhesive tape to remove impurities. Usually, it takes more than 30 tape strip- pings to remove $\mathrm{SC},{ }^{26}$ so the method in this study neither harms the skin nor removes SC. Thus, the animal model can be ready for in vivo experiments directly. For in vivo experiments, ten rats were divided into two groups and topically treated with PEG-400 or the mixture of PEG-400 and Thiazone, respectively. For in vitro skin experiments, fresh samples were obtained from the dorsal skin of the prepared animal models, and then cut to squares of $3 \times 3 \mathrm{~cm}$ after the subcutaneous tissue were removed carefully. In this work, 27 samples were divided into three groups and used for the in vitro experiments. Each group had nine samples that were topically treated by saline, PEG-400, or the mixture of PEG400 and Thiazone, respectively.

\subsection{Spectroscopic Measurement of Skin In Vitro and Data Analysis}

The skin sample was placed between two glass slides, and the thickness of the sample was measured by using a micrometer. A commercial spectrophotometer (Lambda 950, PerkinElmer, Waltham, Massachusetts) with a $150-\mathrm{mm}$ integrating sphere was used for the measurements of diffuse reflectance and diffuse transmittance of the skin sample. First, the spectra of transmittance or reflectance (400 to $1400 \mathrm{~nm}$ ) and the initial thickness were obtained as a reference. Then the chemical agent was topically applied onto the epithelium surface of the sample, and the thickness and spectra were measured at time intervals of $4,12,24$, and $40 \mathrm{~min}$, respectively. To eliminate the effect of mirror reflectance, the solution on the sample was removed with filter paper right before acquiring the spectrum, and added again right after the measurement.

Based on the measurement of spectra and the thickness of the sample, the reduced scattering coefficient and absorption coefficient of skin are calculated with the IAD algorithm. ${ }^{27}$ Furthermore, we calculated the relative changes of the reduced scattering coefficient $\left(\mu_{s}^{\prime}\right)$ at $630 \mathrm{~nm}$ after treatment of the agents:

$$
\frac{\Delta \mu_{s}^{\prime}}{\mu_{s \text { initial }}^{\prime}}=\frac{\mu_{s \text { treated }}^{\prime}-\mu_{s \text { initial }}^{\prime}}{\mu_{s \text { initial }}^{\prime}},
$$

where the subscripts "initial" and "treated" refer to the skin sample before and after the application of chemical agents at the different time intervals, respectively.

Data are given as mean \pm standard deviation. A one-factor analysis of variance (ANOVA) was applied to determine the significant difference for the relative changes in reduced scattering coefficient $(630 \mathrm{~nm})$ caused by saline, PEG-400, and the mixture. The distributions of relative changes were compared with a Kolmogorov-Smirnov test, and the testing for homogeneity of variance was carried out with a $\chi^{2}$ test. The hypothesis employed was that for each group, the relative changes were equal. By the $\mathrm{F}$ test, the hypothesis was rejected. Then the data of the three groups were inspected for differences between pairs of means by the Q method (studentNewman-Keul test). Changes were considered significant if $P<0.05$.

\subsection{Laser Speckle Contrast Imaging for Evaluating the Optical Clearing of Rat Skin In Vivo}

The optical clearing of rat skin in vivo is evaluated by the 


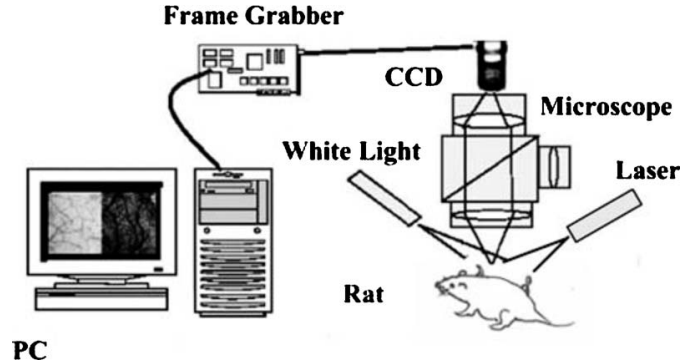

Fig. 1 Laser speckle contrast imaging system.

laser speckle contrast imaging technique. The schematic of the LSCI system is shown in Fig. 1. A He-Ne laser $(\lambda=632.8 \mathrm{~nm}, 10 \mathrm{~mW}$, Melles Griot, Carlsbad, California) is coupled to a fiber with a diameter of $8 \mathrm{~mm}$, and illuminates the interested area evenly at a 45-deg incidence. The illuminated area is imaged through a zoom microscope (SZ6045TR, Olympus, Japan) onto a CCD camera (CoolSNAPes, Roper Scientific, Tuscon, Arizona) with $640 \times 480$ pixels. Each pixel size is $9.9 \times 9.9 \mu \mathrm{m}$, so we can obtain the imaging area of $9 \times 7 \mathrm{~mm}$ to $1 \times 0.8 \mathrm{~mm}$ according to continuously adjustable magnification of 0.7 to 6.3. The CCD exposure time was set at $20 \mathrm{~ms}$. A sequence of laser speckle images reflected from the skin are acquired through PVCAM software ${ }^{28}$ (Roper Scientific, Tucson, Arizona) at $40 \mathrm{~Hz}$, connected to a PC. The LSTCA method (the temporal statistics of light intensity fluctuations caused by moving particles over a number of frames) is applied to calculate the velocity and obtain the 2-D distribution of blood flow. ${ }^{6,7}$ The speckle temporal contrast images can be constructed by calculating the speckle temporal contrast of each image pixel in a time sequence. The value of the speckle temporal contrast $C$ at pixel $(i, j)$ is calculated using Eq. (2) ${ }^{7}$ :

$$
\begin{aligned}
C(i, j) & =\sigma_{i, j} /\left\langle I_{i, j}\right\rangle \\
& =\left(\frac{1}{N-1}\left\{\sum_{n=1}^{N}\left[I_{i, j}(n)-\left\langle I_{i, j}\right\rangle\right]^{2}\right\}\right)^{1 / 2} /\left\langle I_{i, j}\right\rangle,
\end{aligned}
$$

where $I_{i, j}(n)$ is the CCD counts at pixel $(i, j)$ in the $n$ 'th raw speckle map, $N$ is the number of maps acquired, and $\left\langle I_{i, j}\right\rangle$ is the mean value of CCD counts at pixel $(i, j)$ over the $N$ maps.

Cheng et al. indicate that there is a high correlation value $\left(R^{2}=0.96\right)$ between the actual velocity and calculated value when the number of frames is 15 , and it almost keeps constant when the number of frames increases. ${ }^{6}$ Therefore, we select 20 frames $(N=20)$ for laser speckle temporal contrast analysis in this work. When a white light is used instead of the laser, the illuminated area can also be imaged by the CCD and recorded by the computer.

Place the prepared animal model on a constant temperature stage $\left(38^{\circ} \mathrm{C}\right)$, then obtain a set of original photographs and raw speckle maps by switching the light source. After the chemical solution is treated on the surface of the dorsal skin, a set of photographs and raw speckle maps are recorded at 2-min intervals, respectively. After $40 \mathrm{~min}$, apply normal saline to the region of interest, and take the white light image and speckle image again in $2 \mathrm{~min}$.
To compare the morphological differences between the region of interest and surrounding area of skin, pictures were taken with a common digital camera (T200, Sony, Japan) during the intervals.

\section{Results and Analysis}

Even though the mixture of PEG-400 and Thiazone-induced optical clearing of in vitro skin were investigated by Mao et al. ${ }^{19}$ and Jiang et al., ${ }^{20}$ both were conducted on porcine skin. Jiang et al. mixed PEG-400 and Thiazone at a volume ratio of $1: 1$. To illustrate the optical clearing effect of rat skin by topical application of chemical agents, both in vitro and in vivo experiments were performed in this study.

\subsection{Changes in Optical Properties of Rat Skin In Vitro Caused by Different Agents}

\subsubsection{Changes in reflectance and transmittance properties of rat skin}

In general, the changes in reflectance and transmittance of tissue can demonstrate the optical clearing effect. ${ }^{12}$ Figure 2 shows typical changes in reflectance and transmittance spectra after the application of saline, PEG-400, and the mixture of PEG-400 and Thiazone on the rat skin in vitro at time intervals of 0 min (initial state), 12, 24, and $40 \mathrm{~min}$. The results show that saline hardly affects the reflectance and transmittance of rat skin. However, with increasing treatment time of PEG-400 or the mixture, optical transmittance increases and reflectance decreases. Comparing the results of the two agents, we find that the changes caused by the mixture solution are much bigger than those of PEG-400. That is, Thiazone can enhance the optical clearing effect further.

\subsubsection{Changes in reduced scattering and absorption properties of rat skin}

Although OCA treatment can induce increases in transmittance and decreases in reflectance, these changes could be affected by the thickness of the samples. Hence, the changes of tissue optical properties reflect exactly the optical clearing effect induced by different agents. In this work, we calculated the reduced scattering coefficient and absorption spectra from the measurements of reflectance and transmittance spectra with the IAD program. Figure 3 shows typical changes at different treatment time intervals for all agents used.

As shown in the figure, the chemical agents almost induce no influence on the absorption spectra of tissue except at $400 \mathrm{~nm}$. In biological tissue, hemoglobin has a strong absorption at 416 to $420 \mathrm{~nm}$. When OCAs enter into the skin, they can interact with hemoglobin and thus induce the increase of absorption spectra. However, it needs some intensive future study. In contrast, PEG-400 and the mixture can decrease the reduced scattering coefficient significantly, while saline cannot. The longer the OCAs treat, the larger the reduced scattering coefficient decreases.

\subsubsection{Relative changes in reduced scattering coefficient and the statics analysis}

To quantitatively compare the optical clearing effect of in vitro skin samples caused by different agents, we calculate the relative changes of reduced scattering coefficients of skin 


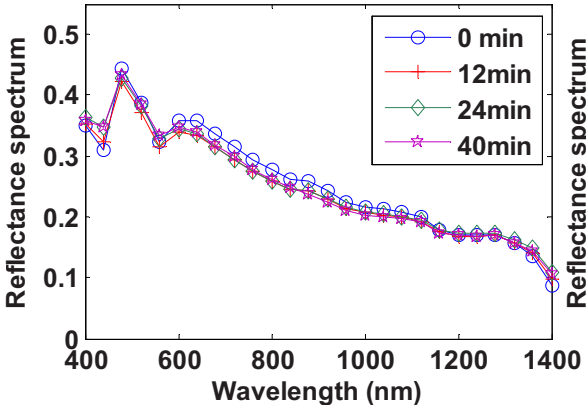

Saline

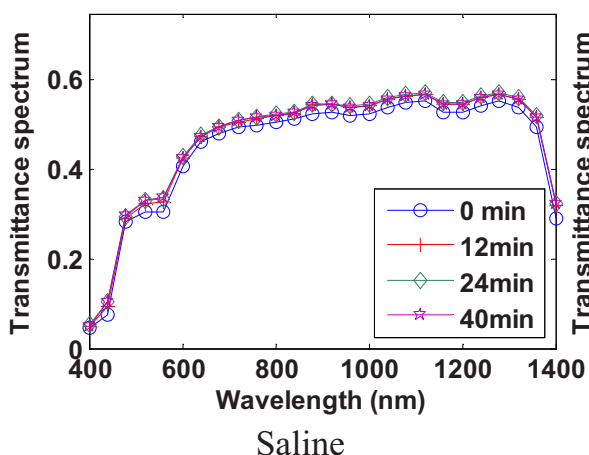

Saline

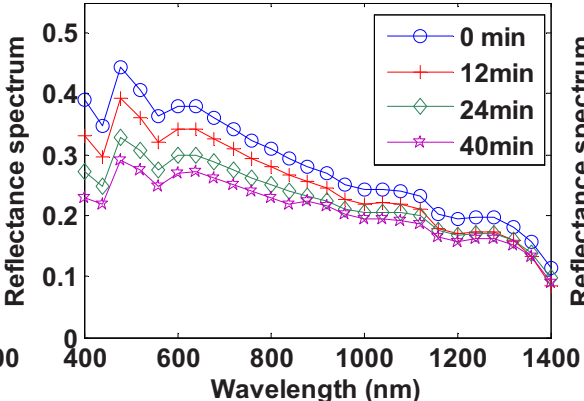

PEG-400

(a)

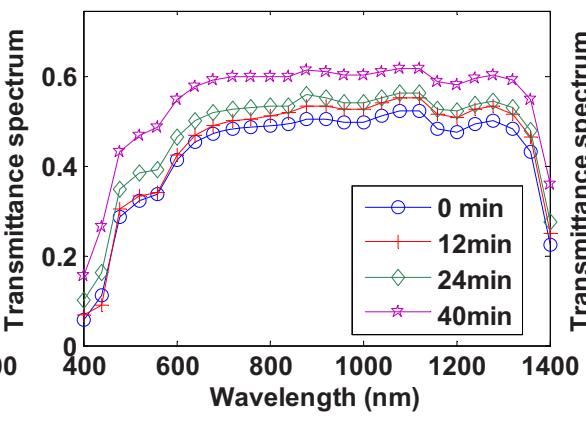

PEG-400

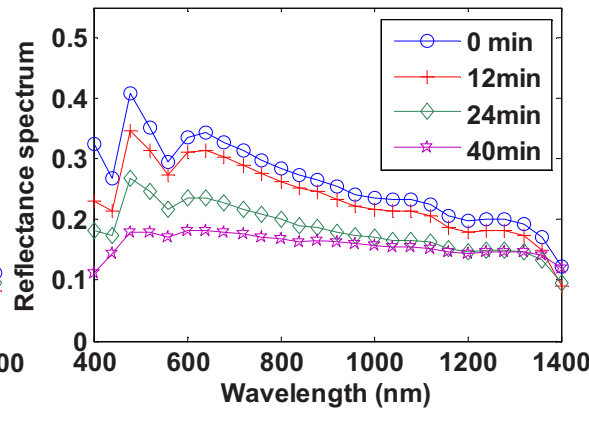

PEG-400+Thiazone

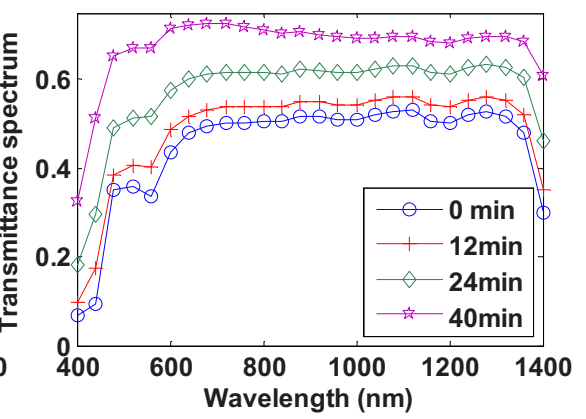

PEG-400+Thiazone

(b)

Fig. 2 Typical spectra of (a) reflectance and (b) transmittance of in vitro skin sample at initial state and different times (12, 24 , and 40 min) after the application of the chemical agents (saline, PEG-400, and a mixture of PEG-400 and Thiazone).

samples at $630 \mathrm{~nm}$ after 40 -min treatment of the agents. An analysis of variance was applied to assay changes caused through treatment of the three agents for statistical differences. The results are summarized in Table 1.

As shown in Table 1, after topical application of saline, PEG-400, or the mixture onto in vitro skin samples, the reduced scattering coefficients decrease to 93,79 , and $64 \%$ of the initial value, respectively. In addition, the relative changes in reduced scattering coefficients are significantly different at the $99 \%$ level between all paired conditions. Thiazone can significantly enhance the penetration of PEG-400 $(P<0.01)$. Compared to saline, PEG-400 alone can also have very significant optical clearing effect for in vitro skin $(P<0.01)$.

\subsection{Accessing the Dermal Blood Vessel and Flow Information through Intact Rat Skin}

The ultimate goal of the optical clearing technique is to serve for clinical applications, but there exists a certain degree of difference in the physiology and metabolism state between in vitro and in vivo skin. Here we show some in vivo experimental results, including white light photographs, taken by a digital camera and velocity maps by LSCI system.

\subsubsection{Visible optical clearing process of in vivo skin}

Figure 4 shows typical morphological pictures of rat skin in vivo at different times after application of PEG-400 and the mixture of PEG-400 and Thiazone. The recovery after 2-min treatment of saline is also shown. At the beginning, the dermal blood vessel is invisible to the naked eye. After having topi- cally applied the mixture of PEG-400 and Thiazone on the rat skin for $4 \mathrm{~min}$, we can observe some large vessels through the intact skin. Up to $12 \mathrm{~min}$, even the small branch vessels can be distinguished clearly, just as the region in the rectangle shows. Moreover, this situation will be allowed to continue. After 40-min treatment of the mixture, we applied the saline onto the interested area. Immediately, the skin is recovered to the initial state and the blood vessels are out of view. By contrast, we can not observe any blood vessel beneath the skin after treatment of PEG-400.

\subsubsection{Laser speckle temporal contrast analysis for evaluating optical clearing of skin in vivo}

The LSCI technique was used to monitor the rectangle area, shown in Fig. 4. The speckle temporal contrast image was constructed by calculating the speckle temporal contrast of each pixel in the time sequence. Figure 5 shows typical photographs and the speckle temporal contrast maps of skin at initial state, and 4, 12, 24, and 40 min after treatment with PEG-400, the mixture of Thiazone and PEG-400, and 2 min after treatment with saline.

The photographs in the top row show the visual optical clearing process of skin, and the temporal contrast maps in the bottom row are quite consistent with the photographs shown in the top row. We find that the image quality becomes better by topical application of the mixture, and more details of the dermal blood vessels can be seen. The temporal contrast maps demonstrate that the topical application of the mixture can decrease the temporal contrast of the vessels, which provides 


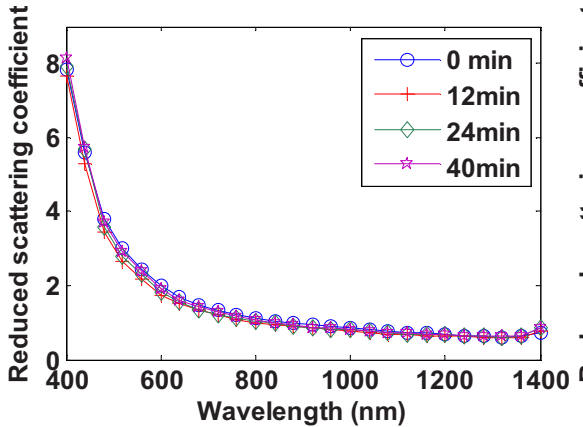

Saline

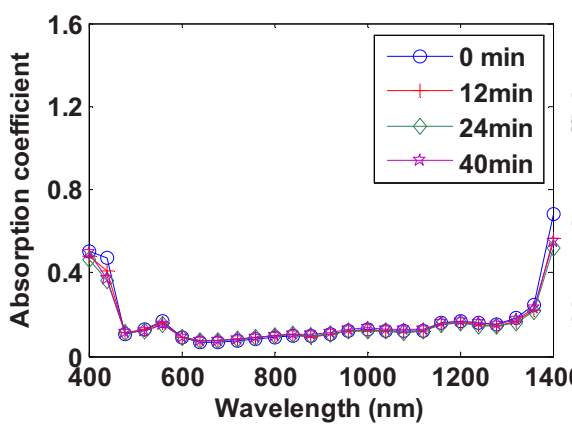

Saline

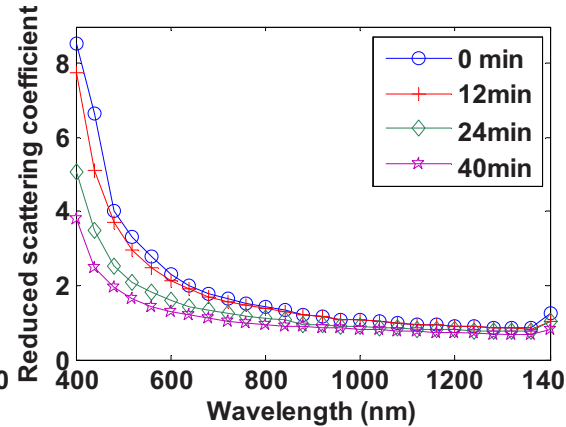

PEG-400

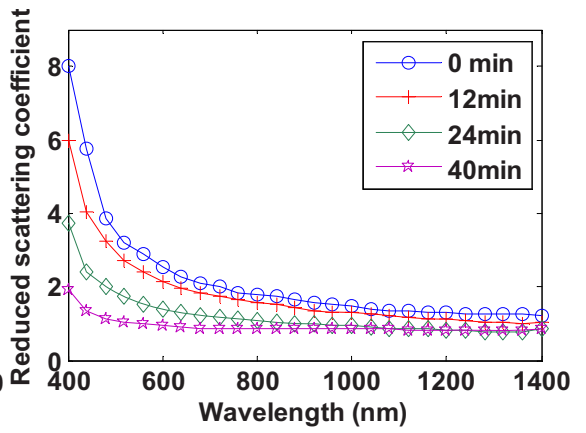

PEG-400+Thiazone

(a)

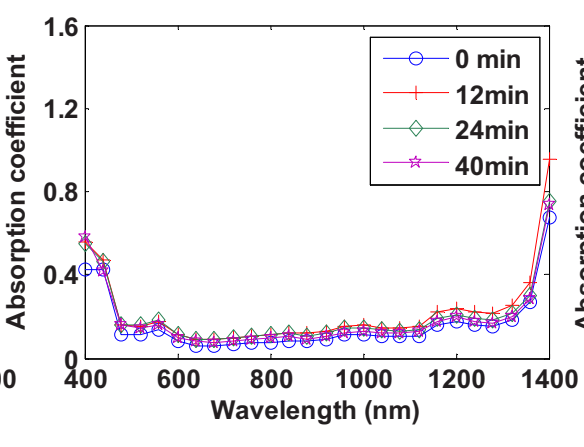

PEG-400

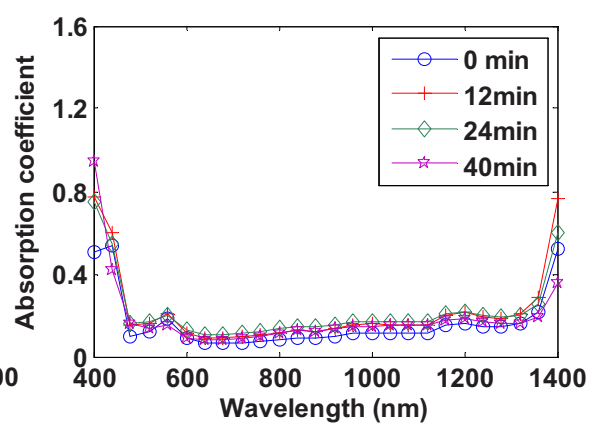

PEG-400+Thiazone

(b)

Fig. 3 Typical spectra of (a) reduced scattering and (b) absorption of in vitro skin sample at initial state and different times (12, 24 , and 40 min) after the application of the chemical agents (saline, PEG-400, and a mixture of PEG-400 and Thiazone).

better visualization of the blood flow. The treatment of saline can make the dermal vessels invisible very quickly, and then the contrast can be recovered. In contrast with the prior description, after having been treated with pure PEG-400, only main vessels can be distinguished faintly from the temporal contrast maps, and no branch vessels can be detected. The results are in accordance with that in Fig. 4.

\subsection{Laser Speckle Temporal Contrast Analysis for Evaluating Optical Clearing of Skin in vivo Quantitatively}

In particular, the relative changes of temporal contrast in areas shown in Fig. 5 are plotted in Fig. 6. Here, 1, 3, and 4 represent three areas of two main vessels and a branch vessel, respectively, while 2 and 5 represent the areas where there is no blood vessel. The results show that the temporal contrast goes down quickly during the first $12 \mathrm{~min}$, and then keeps constant with a small vibration for 12 to $40 \mathrm{~min}$. For the imaging area treated by the mixture, the changes in trends of temporal contrast between blood vessels 3 and 4 are identical, and the minimal temporal contrast at the areas of the main blood vessel and branch blood vessel reduces 80 and $75 \%$, respectively. There is slight increase in the temporal contrast for 12 to $40 \mathrm{~min}$. This may attribute to the metabolism of biological tissue. For in vivo rat, when the chemical agents immerse into dermis, the local skin shows optical clearing. However, metabolism takes the agents away, so the optical clearing effect may reduce with passing time, thus the normal-

Table 1 The relative changes of reduced scattering coefficient at $630 \mathrm{~nm}$ after 40 -min treatment of different agents. ${ }^{* *} P<0.01$ for difference between relative changes caused by treatment of different agents.

\begin{tabular}{lcccc} 
& & & \multicolumn{2}{c}{ Difference $(\alpha=0.05)$} \\
\cline { 4 - 5 } Chemical agents & sample & $R C_{i}\left(\frac{\Delta \mu_{s}^{\prime}}{\mu_{s}^{\prime} \text { initial }}\right)$ & $R C_{i}-R C_{i, \text { saline }}$ & $R C_{i}-R C_{i, \mathrm{PEG}-400}$ \\
\cline { 4 - 5 } Saline & 9 & $-0.07 \pm 0.03$ & - & - \\
PEG-400 & 9 & $-0.21 \pm 0.06$ & $-0.14^{* *}$ & - \\
PEG-400 + Thiazone & 9 & $-0.36 \pm 0.06$ & $-0.29^{* *}$ & $-0.15^{* *}$ \\
\hline
\end{tabular}




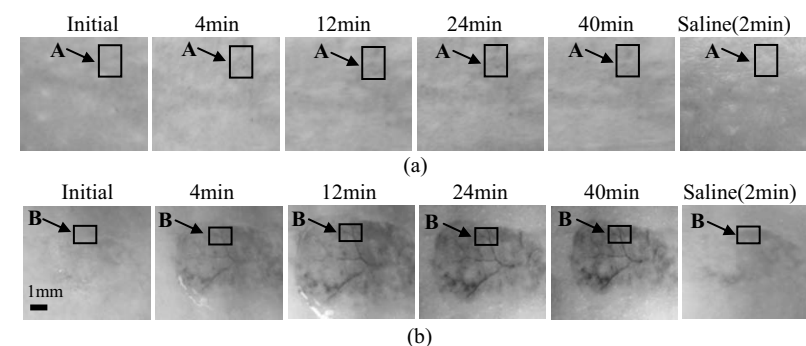

Fig. 4 Visible optical clearing process of in vivo rat skin before and after treatment with (a) PEG-400 and (b) mixed solution of PEG-400 and Thiazone on skin surface. The rectangles (A and B) indicate the interest areas.

ized contrast increases gradually for 12 to $40 \mathrm{~min}$. Nevertheless, with treatment of PEG-400, the minimal temporal contrast at the area of vessel 1 only reduces $40 \%$, and information of the branch vessel cannot be detected at all. At areas where there is no blood vessel, the decline of temporal contrast for no blood vessel areas is much less than that for vessel areas after treatment of the mixture or PEG- 400 .

The results show that there are vibrations of the contrast on all skin sites. It is the reason that OCAs immersed into skin make the local area transparent. The reduction in scattering of skin decreases the static speckle and the contrast of the local area. In blood vessel areas, both static and dynamic speckle contribute to the calculation of temporal contrast. The reduction of the static speckle increases the ratio of dynamical speckle of the area, and more useful information is analyzed. However, in areas where there are no blood vessels, the temporal contrast is due to the static speckle, and as a result, the temporal contrast also changes slightly with treatment of OCAs or saline.

\section{Discussions and Conclusions}

The results indicate that the topical application of Thiazone and PEG-400 can enhance light penetration in skin, which enables LSCI to access the dermal blood flow by a noninvasive method. The mechanism of the optical clearing of skin
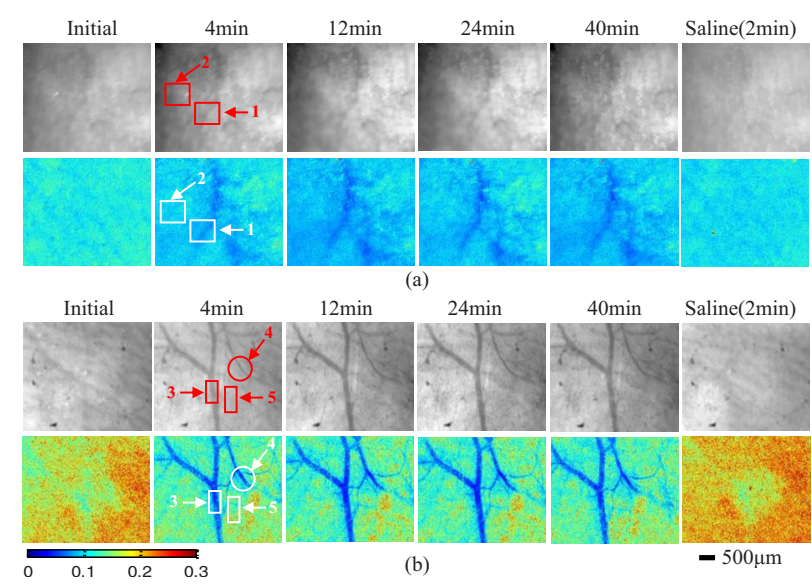

Fig. 5 Photographs (top row) and temporal contrast maps (bottom row) of rectangle areas in Fig. 4 at initial state, 4, 12, 24, 40 min after treatment of different OCAs, and 2 min after treatment of saline. (a) PEG-400, (b) mixed solution of PEG-400 and Thiazone.

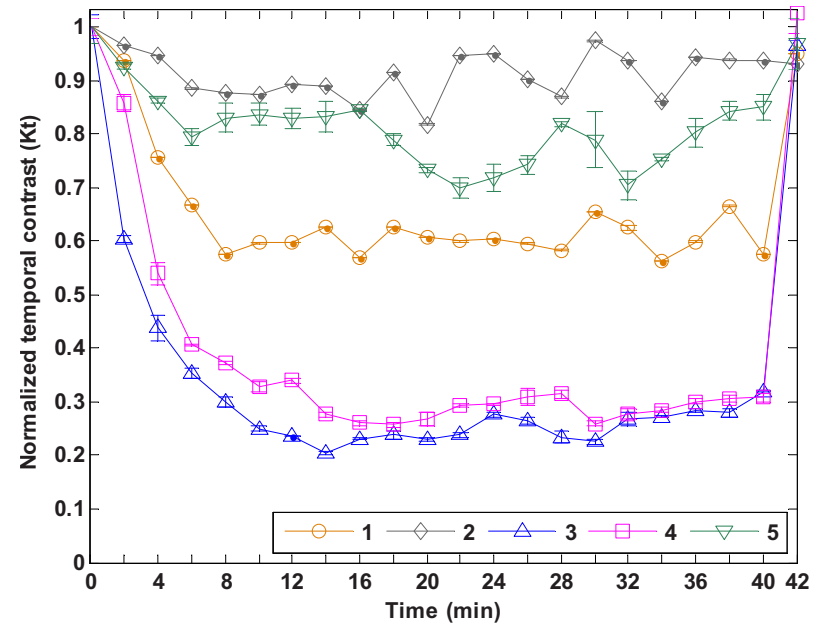

Fig. 6 Dynamic temporal contrast in five specific areas 1, 2 [Fig. 5(a), treatment with PEG-400], 3, 4, and 5 [Fig. 5(b), treatment with mixture of PEG-400 and Thiazone] before and after the application of optical clearing agents and saline. Here, 1, 3, and 4 are vessel areas, while 2 and 5 are no-vessel areas.

may attribute to the newly applied chemical penetration enhancer, Thiazone, which is a derivative of Azone, but has a penetration enhancing effect two times higher. ${ }^{24}$ The possible mechanism of Thiazone would be that the fluidity of the hydrophobic stratum corneum regions is increased and the permeation resistance of the horny layer against drug substances is reduced by them, ${ }^{29}$ which enhances the permeability of PEG-400 from surface to dermis and makes the concentration of PEG-400 in dermis increase. PEG-400 with high refractive index improves the matching of refractive indices between the scatters and interstitial fluid in dermis, and then reduces the scattering of skin. The flush of normal saline on transparent skin can make it recover. The probable reason is that a large quantity of saline penetrates through the gap of epidermal cells into the dermis, and results in the transparent skin becoming turbid again.

Skin is a high scattering media. In general, the dynamic speckle signal from the blood flow is almost concealed by the strong static speckle signal from the skin. Even though the LSTCA method can decrease the effect of stationary speckles, it is still difficult to obtain information about dermal blood flow. However, this situation would be changed if the optical clearing technique was applied, because the optical clearing method can reduce the scattering of skin and make the skin transparent. Thus, more dynamic speckle signal from the area of blood flow can penetrate through the transparent skin and be detected by the CCD. As a consequence, improvement of image quality has permitted us to access the structure and flow information of dermal blood vessels with the LSCI technique.

Briefly, topical application of the penetration enhancer Thiazone with PEG-400 onto the surface of rat skin in vitro can reduce scattering to $60 \%$ of the initial value; and on rat epidermis in vivo, can make the skin transparent. This optical clearing method enables us to observe dermal blood vessels and image dermal blood flow through intact rat skin by the LSCI technique. Furthermore, by combining this method with the intrinsic optical imaging technique, ${ }^{30}$ we can access the 
metabolic oxygen information of skin microcirculation. It is very important to diagnose peripheral vascular disease based on subcutaneous microcirculation. This method combined with other optical imaging techniques, such as fluorescence imaging, ${ }^{31}$ microscopy imaging, ${ }^{32}$ etc., will enhance the capabilities of noninvasive optical diagnosis for other diseases. Also, with certain techniques, the pharmacological effect of some kind of vascular drugs can also be evaluated.

\section{Acknowledgments}

This study was supported by the National Nature Science Foundation (grant numbers 30770552 and 30911120074 ), and the National High-Tech Research and Development Program (number 2008AA02Z107) of China. The authors would like to thank Pengcheng Li for his help on the LSCI technique, and Shaoqun Zeng for his helpful discussions. We also appreciate Valery V. Tuchin for his help.

\section{References}

1. M. Rossi and A. Carpi, "Skin microcirculation in peripheral arterial obliterative disease," Biomed. Pharmacother 58, 427-431 (2004).

2. J. D. Briers and S. Webster, "Laser speckle contrast analysis (LASCA): a non-scanning, full-field technique for monitoring capillary blood flow," J. Biomed. Opt. 1, 174-79 (1996).

3. J. D. Briers, G. Richards, and X. W. He, "Capillary blood flow monitoring using laser speckle contrast analysis (LASCA)," J. Biomed. Opt. 4(1), 164-175 (1999).

4. J. D. Briers, "Laser Doppler, speckle and related techniques for blood perfusion mapping and imaging," Physiol. Meas 22, R35-R66 (2001).

5. S. S. Liu, P. Li, and Q. Luo, "Fast blood flow visualization of highresolution laser speckle imaging data using graphics processing unit," Opt. Express 16(19), 14321-14329 (2008).

6. H. Cheng, Q. Luo, S. Zeng, S. Cheng, J. Cen, and H. Gong, "Modified laser speckle imaging method with improved spatial resolution," J. Biomed. Opt. 8(3), 559-564 (2003).

7. P. Li, S. Ni, L. Zhang, S. Zeng, and Q. Luo, "Imaging cerebral blood flow through the intact rat skull," Opt. Lett. 31, 1824-1826 (2006).

8. H. Cheng, Q. Luo, S. Zeng, S. Chen, W. Luo, and H. Gong, "Hyperosmotic chemical agent's effect on in vivo cerebral blood flow revealed by laser speckle," Appl. Opt. 43(31), 5772-5777 (2004).

9. D. Zhu, W. Lu, Y. Weng, H. Cui, and Q. Luo, "Monitoring thermal induced changes in tumor blood flow and micro-vessels with laser speckle contrast imaging," Appl. Opt. 46, 1911-1917 (2007).

10. W. Redisch, E. J. Messina, G. Hughes, and C. Mcewen, "Capillaroscopic observations in rheumatic diseases," Ann. Rheum. Dis. 29, 244-253 (1970).

11. V. V. Tuchin, I. L. Maksimova, D. A. Zimnyakov, I. L. Kon, A. H. Mavlutov, and A. A. Mishin, "Light propagation in tissues with controlled optical properties," J. Biomed. Opt. 2(4), 401-417 (1997).

12. V. V. Tuchin, "Optical Immersion as a new tool for controlling optical properties of tissues and blood," Laser Phys. 15, 1109-1136 (2005).

13. V. V. Tuchin, G. B. Altshuler, A. A. Gavrilova, A. B. Pravdin, D. Tabatadze, J. Childs, and I. V. Yaroslavsky, "Optical clearing of skin using flashlamp-induced enhancement of epidermal permeability,"
Lasers Surg. Med. 38, 824-836 (2006).

14. X. Xu, Q. Zhu, and C. Sun, "Combined effect of ultrasound-SLS on skin optical clearing," IEEE Photonics Technol. Lett. 20(24), 21172119 (2008).

15. O. Stumpp, B. Chen, and A. J. Welch, "Using sandpaper for noninvasive transepidermal optical skin clearing agent delivery," $J$. Biomed. Opt. 11(4), 041118 (2006).

16. J. Yoon, T. Son, E. Choi, B. Choi, J. S. Nelson, and B. Jung, "Enhancement of optical skin clearing efficacy using a microneedle roller," J. Biomed. Opt. 13(2), 021103 (2008).

17. J. Jiang and R. K. Wang, "Comparing the synergistic effects of oleic acid and dimethyl sulfoxide as vehicles for optical clearing of skin tissue in vitro," Phys. Med. Biol. 49, 5283-5294 (2004).

18. X. Xu and Q. Zhu, "Evaluation of skin optical clearing enhancement with Azone as a penetration enhancer," Opt. Commun. 279, 223-228 (2007).

19. Z. Mao, Y. Hu, Y. Zheng, W. Lu, Q. Luo, and D. Zhu, "Experimental study on influence of Thiazone on optical clearing of piglet skin in vitro," Acta Opt. Sin. 27(3), 526-530 (2007) (in Chinese).

20. J. Jiang, W. Chen, R. K. Wang, and K. Xu, "Availability of thiazone as an enhancer for optical clearing of skin tissue in vitro," Proc. SPIE 6858, 68580N (2008).

21. G. Vargas, J. K. Barton, and A. J. Welch, "Use of hyperosmotic chemical agent to improve the laser treatment of cutaneous vascular lesions," J. Biomed. Opt. 13(2), 021114 (2008).

22. G. Vargas, A. Readinger, S. S. Dozier, and A. J. Welch, "Morphological changes in blood vessels produced by hyperosmotic agents and measured by optical coherence tomography," Photochem. Photobiol. 77(5), 541-549 (2003).

23. B. Choi, N. M. Kang, and J. S. Nelson, "Laser speckle imaging for monitoring blood flow dynamics in the in vivo rodent dorsal skin fold model," Microvasc. Res. 68, 143-146 (2004).

24. L. Xiong, "A new skin penetration promoter: thiazone," Fine Specialty Chem. 12(21), 9-11 (2004) (in Chinese).

25. L. Xiong, Z. Kong, and X. Wang, "The application of $\mathrm{N}$-alkylbenzisothiazolone as a penetration enhancer," Patent CN00107448.2 (2000).

26. H. Pinkus, "Examination of the epidermis by the strip method of removing horny layers. I. Observations on thickness of the horny layer, and on mitotic activity after stripping," J. Invest. Dermatol. $\mathbf{1 6}$, 383-386 (1951).

27. S. Prahl, M. van Gemert, and A. Welch, "Determining the optical properties of turbid media by using the adding doubling method," Appl. Opt. 32, 559-568 (1993).

28. See http://www.princetoninstruments.com/products/software.aspx\#5.

29. J. Kalbitz, R. Neubert, and W. Wohlrab, "Modulation of drug penetration in the skin," Pharmazie 51, 619-637 (1996).

30. P. Li, Q. Luo, W. Luo, S. Cheng, S. Zeng, and Q. Luo, "Spatiotemporal characteristics of cerebral blood volume changes in rat somatosensory cortex evoked by sciatic nerve stimulation and obtained by optical imaging," J. Biomed. Opt. 8(4), 629-635 (2003).

31. R. Cicchi, F. S. Pavone, D. Massi, and D. D. Sampson, "Contrast and depth enhancement in two-photon microscopy of human skin ex vivo by use of optical clearing agents," Opt. Express 13(7), 2337-2344 (2005).

32. S. Zeng, X. Lv, C. Zhan, W. R. Chen, W. Xiong, S. L. Jacuqes, and Q. Luo, "Simultaneous compensation for spatial and temporal dispersion of acousto-optical deflectors for two-dimensional scanning with a single prism," Opt. Lett. 31(8), 1091-1093 (2006). 